biological (51\%), articular (38\%), haematological (24\%) and glandular (22\%). Low DAS was reported in $4480(56 \%)$ patients, moderate DAS in $2483(31 \%)$ and high DAS in 1098 (14\%) patients. The mean baseline ESSDAI was higher the younger the patient was $(p<0.001)$, higher in White patients ( 6.9 vs $5.1, p<0.001)$, males ( 8.4 vs $6.2, p<0.001)$, those with positive ocular ( 6.7 vs $4.9, p<0.001)$ or oral $(6.8$ vs $6.2, p=0.016$ ) tests, and those with ANA (6.9 vs $4.5, p<0.001$ ), RF (7.5 vs 5.8 , $\mathrm{p}<0.001)$ and anti-Ro/La antibodies (7.2 vs $4.4, \mathrm{p}<0.001)$. Logistic regression identified as independent variables White ethnicity (OR 3.07), abnormal ocular tests (OR 2.14), ANA (OR 1.67) and Ro/La autoantibodies (OR 2.78).

Conclusions: This is the largest series of patients with primary SS in whom the ESSDAI score has been evaluated. Primary SS is undeniably a systemic disease even at the time of diagnosis, with nearly $80 \%$ of patients showing an ESSDAI score $>0$

Disclosure of Interest: None declared

DOI: 10.1136/annrheumdis-2017-eular.4472

\section{THU0682 POPGEN-OSSA: DEVELOPMENT OF AN ORGAN SPECIFIC SELF ASSESSMENT (OSSA) FOR INTERDISCIPLINARY DOCUMENTATION OF PATIENT REPORTED CLINICAL OUTCOMES}

R. Zeuner ${ }^{1}$, U. Gsell ${ }^{2}$, M. Hübenthal ${ }^{3}$, S. Schreiber ${ }^{4}$, A. Franke ${ }^{3}$, M. Laudes ${ }^{4}$ J.O. Schröder ${ }^{1} .{ }^{1}$ Section of Rheumatology, Clinic for Internal Medicine I, University Hospital Schleswig-Holstein, Campus Kiel, Kiel; ${ }^{2}$ Klinik Eichstätt, Eichstätt; ${ }^{3}$ Institute of Clinical Molecular Biology; ${ }^{4}$ Clinic for Internal Medicine I, University Hospital Schleswig-Holstein, Campus Kiel, Kiel, Germany

Background: Patient reported outcome measures are comprised of either sets of questionnaires or patient global assessment based on visual analogue scale (VAS). These patient-reported outcome measures lack accuracy and/or clinical feasibility when comparing heterogeneous patient groups with different diseases, or when characterizing patients with systemic disease involving different organ systems.

Objectives: Developing a clinical feasible patient-reported outcome measure based VAS assessment of different organ systems.

Methods: Patients were asked to rate their health status in a $10 \mathrm{~cm}$ VAS $(0-100 \%)$ concerning their global health as well as of different organ systems, namely heart, lung, muscle and joints, gastro-intestinal, metabolic, uro-genital, skin, neuro-psychiatric, eyes and ears. All VA-scales were "anchored". Patients were advised to rate their health status below $75 \%$ if they felt "medical action is needed", they should rate the health status $<50 \%$ in case of a "strong need for medical action" and $<25 \%$ in case of a "medical emergency".

336 patients from different outpatient clinics (cardiologic, pneumologic, gastrointestinal, nephrologic, neurologic, dermatologic, rheumatologic, ophthalmologic and obesity outpatient clinic) as well as patients from internal emergency clinics and a general practitioner clinic were evaluated. Both, patients and the attending physicians completed the Popgen-OSSA. In addition the attending physician was asked to document ranking of the 5 most important diagnoses of the patient. Statistical analysis was carried out using non-parametric testing. Furthermore, to predict main diagnoses based on patients's as well as physician's OSSA state-of-the-art machine learning tools, namely support vector machines (SVMs), were applied. To assess model performance multi-class AUC (area under the ROC curve) according to Hand and Till (2001) was estimated based on repeated cross validation ( 10 folds, 5 repeats), optimizing the SVM's hyperparamters using grid search.

Results: The test showed a good reproducibility. With a mean percentage of $74 \pm 0.98 \mathrm{SE}$ and $66 \pm 1.17 \mathrm{SE}$, respectively, the physicians OSSA rating was significantly higher than the rating of the patients $\left(p_{\text {wilcoxon }}<0.001\right)$. Models predicting main diagnoses were constructed and estimated to perform with multi-class AUCs of $63.5 \%$ and $73.4 \%$ based on patient's and physician's OSSA, respectively.

Conclusions: In this preliminary trial with low sample size the Popgen-OSSA showed a good reproducibility and allowed a correct allocation of the patient's clinical problem to involved organ system by SVM analysis with multi-class AUC of up to $73.4 \%$. These data merit further investigation and development of the Popgen-OSSA on larger patient cohorts.

\section{References:}

[1] David J. Hand and Robert J. Till (2001). A Simple Generalisation of the Area Under the ROC Curve for Multiple Class Classification Problems. Machine Learning 45(2), p. 171-186. DOI: 10.1023/A:1010920819831.

Disclosure of Interest: R. Zeuner Grant/research support from: Pfizer, Novartis, UCB, U. Gsell: None declared, M. Hübenthal: None declared, S. Schreiber: None declared, A. Franke: None declared, M. Laudes Grant/research support from: Roche, Lilly, MSD, J. Schröder: None declared

DOI: 10.1136/annrheumdis-2017-eular.4325

\section{THU0683 RAPID DETERMINATION OF THE INFLAMMATION MARKER CALPROTECTIN IN SERUM FROM PATIENTS WITH INFLAMMATORY ARTHRITIS AT THE POINT OF CARE}

N. Ryter ${ }^{1}$, A. Szentpetery ${ }^{2}$, S.R. Pennington ${ }^{3}$, R. Cotti $^{1}$, O. FitzGerald ${ }^{2}$, J.M. Weber ${ }^{1}$. ${ }^{1}$ BÜHLMANN Laboratories AG, Schoenenbuch, Switzerland; ${ }^{2}$ St Vincent's University Hospital; ${ }^{3}$ University College, Dublin, Ireland

Background: A Treat-to-Target (T2T) strategy for inflammatory arthritis, targeting remission or minimal disease activity, is the recommended treatment approach by EULAR and ACR. This strategy relies on "tight monitoring" which necessitates regular clinical examination and measuring acute-phase reactants such as C-reactive protein. Calprotectin (MRP8/14; S100A8/A9), a relatively novel inflammation and disease activity marker in the arthritis field, exhibits several features which fit the "theranostic needs" for accurate therapy monitoring. Those features include discrimination between responders and non-responders [1], detection of subclinical disease activity [2] and prediction of relapse or radiographic progression [3]. The classical method to determine calprotectin in serum (SCAL) is ELISA which is used in service or central laboratories. A rapid and simple determination of SCAL at the point of care is a substantial step forward in supporting clinicians to deliver an efficient T2T strategy. Here, we report on the validation of a quantitative, rapid test which can measure SCAL within 15 minutes.

Objectives: (1) To demonstrate the performance evaluation of a quantitative lateral flow assay combined with a dedicated test reading device for the rapid quantification of calprotectin in serum; and (2) to compare results to a well-established laboratory reference method using patient samples.

Methods: The Quantum Blue ${ }^{\circledR}$ SCAL sandwich lateral flow immunoassay uses two highly specific monoclonal antibodies immobilized on the test membrane and on the gold label. $10 \mu \mathrm{L}$ of serum was diluted in $90 \mu \mathrm{L}$ of chase buffer, $60 \mu \mathrm{L}$ of this mixture was applied onto the lateral flow test cassette, which was incubated for 12 minutes at ambient temperature and then measured with the BÜHLMANN Quantum Blue ${ }^{\circledR}$ Reader. Performance evaluation (sensitivity, linearity, high-dose hook effect, interferences) was carried out according to CLSI guidelines. A method comparison based on 178 serum samples from RA and PsA patients was performed against the BÜHLMANN sCAL (MRP8/14) reference ELISA.

Results: The linearity study over the complete measuring range together with the observed limit of quantification (LoQ) of $<0.5 \mu \mathrm{g} / \mathrm{mL}$ allowed a quantitative measurement in the clinically relevant range from 0.5 to $10.0 \mu \mathrm{g} / \mathrm{mL}$ calprotectin. No high dose hook effect was observed up to a concentration of $200 \mu \mathrm{g} / \mathrm{mL}$. Moreover, no interferences were detected with triglycerides $(37 \mathrm{mmol} / \mathrm{L})$, conjugated bilirubin $(342 \mu \mathrm{mol} / \mathrm{L})$, unconjugated bilirubin $(342 \mu \mathrm{mol} / \mathrm{L})$, and hemoglobin $(200 \mathrm{mg} / \mathrm{dL})$. The Quantum Blue ${ }^{\circledR} \mathrm{SCAL}$ lateral flow assay showed an excellent linear correlation $(r=0.94$, slope $=1.05)$ to the BÜHLMANN SCAL (MRP8/14) reference ELISA. There was a negligible bias of $-3.1 \%$ by Bland-Altman difference plot between the sCAL lateral flow assay and ELISA.

Conclusions: Rapid quantification of serum calprotectin using the Quantum Blue ${ }^{\circledR}$ SCAL assay represents a fast and reliable method for the determination of inflammation and the disease activity of a patient with inflammatory arthritis at the point of care. This rapid test shows excellent agreement to a corresponding laboratory reference method.

References:

[1] Anink J. Arthritis Res Ther (2015);15:200.

[2] Inciarte-Mundo J. Arthritis Res Ther (2016);18:160.

[3] Hurnakova J. Arthritis Res Ther (2015);17:252.

Disclosure of Interest: N. Ryter Employee of: Employee of BÜHLMANN Laboratories AG, A. Szentpetery: None declared, S. Pennington: None declared, R. Cotti Employee of: Employee of BÜHLMANN Laboratories AG, O. FitzGerald: None declared, J. Weber Employee of: Employee of BÜHLMANN Laboratories AG DOI: 10.1136/annrheumdis-2017-eular.4722

\section{THU0684 WORK IMPACT IN AXIAL SPONDYLOARTHRITIS: THE AS-WIS QUESTIONNAIRE PREDICTS THE RISK OF WORK IMPACT: A LONGITUDINAL STUDY OF 101 PATIENTS}

S. Dadoun $^{1}$, C. Jacquemin ${ }^{1}$, S. Kreis ${ }^{1}$, S. Fabre ${ }^{1}$, C. Rein ${ }^{1}$, C. Hudry ${ }^{2}$ E. Pertuiset ${ }^{1}$, B. Fautrel ${ }^{1}$, L. Gossec ${ }^{1} .{ }^{1}$ Rheumatology, Pitié Salpétrière Hospital; ${ }^{2}$ Rheumatology, Cochin Hospital, Paris, France

Background: Axial spondyloarthritis (axSpA) mainly affects the working-age subject and can have a moderate (short-term sick leave) or significant (long-term sick leave, disability status, unemployment) work impact.

Objectives: To evaluate the value of a simple and short (2 minutes) questionnaire, the AS-Work instability scale (AS-WIS) to predict the work impact of axSpA after 1-2 years.

Methods: Longitudinal study in 3 centers in Paris, France. Patients with axSpA according to the rheumatologist and the ASAS criteria were included. Patients were asked twice (1 to 2 year interval) to answer questionnaires evaluating: disease activity, demographic characteristics, impact on work (short-term and long-term sick leave, disability, unemployment), and the ASWIS questionnaire (1): a 20 item, simple screening tool for Work Instability (the consequences of a mis-match between an individual's functional ability and their work tasks). The risk of disability is assessed as low if the score is $<11$, medium between 11-18 and high $>18$. Only patients who answered both questionnaires were included for analyses. Statistical analyses included descriptive analyses and univariate/ multivariate 